\title{
Optimal Design of Underground Garage Sign System in Chengdu New Century Global Center
}

\author{
Qian $\mathrm{Li}^{1}$ \\ ${ }^{1}$ Sichuan Agricultural University, Chengdu, Sichuan Province, China
}

Keywords: Chengdu New Century Global Center; underground garage; sign system; theme; regional culture.

\begin{abstract}
This paper analyzes and studies the underground garage sign system of Chengdu New Century Global Center. Based on field investigation and analysis on the present situation of this underground garage, optimal design for the sign system of underground garage is provided. Combined with advantages and disadvantages of underground garage sign system and Chengdu's local culture, the design concept of "the matization" is put forward. Optimal design for the underground garage sign system of Chengdu New Century Global Center is proposed together with corresponding reforming schemes in detail, hoping to provide some practical suggestions on the design of underground garage sign system in China.
\end{abstract}

\section{Introduction}

The development of underground garage is accompanied by economic development and the increasingly shortage of urban land. [1] With the continuous improvement of people's material life, underground garage has become one of the most important means of parking. Sign system is important in providing information for drivers in the underground parking place which is lack of direction-sense. An excellent sign system can guide drivers to enter and leave the garage quickly and easily, reflect the overall quality of citizens, and represent the historical and cultural heritage of the city. This paper selects the underground garage sign system of Chengdu New Century Global Center as the research object. This garage belongs to large underground garage with typicality and representativeness and can accommodate a large number of vehicles. It can influence the whole southwest area of China, and display the image of Sichuan province. Through researches based on city image design and other theories, it is found that the current underground garage sign system ignore the law of humans' short term memory. Meanwhile, the disordered routes cannot effectively guide drivers into their parking spaces. Thus, the design concept of "the matization" is put forward together with Chengdu regional culture to provide an optimal design for sign system.

\section{Current Situation of Sign System in Chengdu New Century Global Center Underground Garage}

The area is located in Dayuan group, Southern District of Chengdu. It is situated in Tianfu Avenue of Chengdu high-tech Zone and the junction of express highway circling the city. The center covers an area of about 1300 acres; the total construction area is about 1 million 760 thousand square meters; the height is about 100 meters. It is a multi-functional building which contains the central entertainment district, hotels, commercial area, office space and other places. As the largest single building in Asia, it is the largest commercial complex in the new town of Tianfu. It has 5400parking spaces in underground garage, 5600 parking spaces on the ground, and 2800 temporary parking spaces. The garage is in irregular form, and the sign system plays an important guiding role in the underground parking system. A simple and easy understandable sign system can facilitate drivers to enter the parking space, park their cars and leave the garage. Through the investigation of underground garage in Chengdu New Century Global Center, main problems on its sign system are found as following. 
Not unified style. Sign system should realize the function of transmitting information in a unified style. But the design of underground garage in Chengdu New Century Global Center makes guidance information difficult to distinguish. When users select information, false judgment occurs easily.

Mixed color. So many kinds of colors are used to divide regions. In an area which only has less than 20 parking spaces, multiple colors exist at the same time (Figure 1). These similar colors simply cover large is as to divide regions make people difficult to clearly find their parking information in the confined space of underground garage

Obscure partition figures. This garage belongs to large underground garage. It has total 8 partitions; each partition has more than three entrances. English, numbers, various marine animal patterns and diversified section name are used to sign different partitions. People usually choose to remember one or two information; the fuzzy pattern information is easily to be ignored.

Guiding information on the ground is not consistent with underground garage sign system. In the guidance system of the business center, the color of different regions in the planar graph of underground garage is not consistent with colors actually painted in the underground garage. It is easy to mislead users.

Unreasonable positions of indicating information. The sizes, placement and layout of signs in the underground garage make people easy to miss useful instruction information, and choose the wrong direction or get lost. Underground sign system should provide concise information to help drivers quickly identify their location and direction. However, the existing sign system provides complex information, and drivers are difficult to make correct judgment when driving vehicles. These problems directly affect people's experience in the underground parking lot. Under the premise of ensuring the guidance function of sign system, designers should also attach importance to the coordination of modeling, layout and material with the environment, in order to enhance the whole image of the environment. [2]

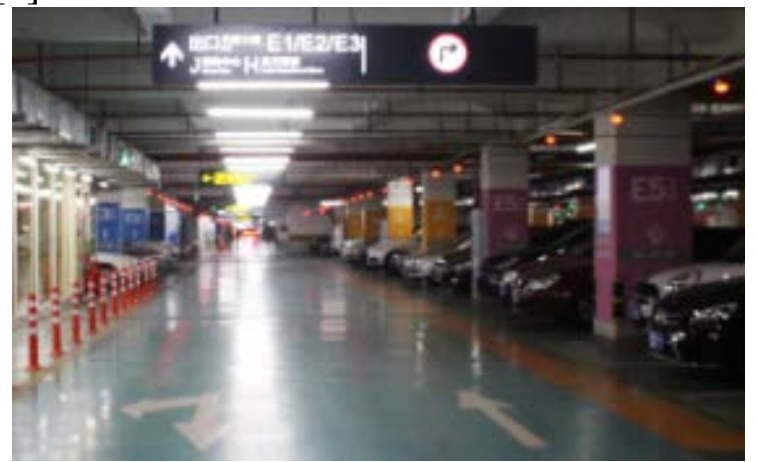

Fig.1 Current situation of sign system in underground garage of Chengdu New Century Global Center

\section{Optimal design Strategies for Underground Garage of Chengdu New Century Global Center}

"The matization" design conception. The dialogue between human behavior and space is the process of visual guidance, or the process of transmitting and receiving spatial information.[3] Johannes Itten, a chromatist, believes that colors should be considered together with graphics. People are easy to thoroughly understand clear geometric figures. [4] For colors and figures, people select what they want to see. Single color information which related to parking information is not easy to remember, since figures and colors are integrated with each other. Thus, the "the matization" design conception is provided, for users need to combine information on their own parking spaces with unique theme figures. The representative regional culture is chosen as the source of themes; specific figures and colors are used to divide different partitions. According to the specific theme of each partition, theme figures are used in the tops, grounds, walls, columns and various facilities in the underground garage. The space is extended in that way. In elevators and other places, more guidance signs should be provided to enhance users' awareness of their positions in the garage, deepen their overall understanding of the underground parking space and the unknown commercial space on the ground, and stimulate their visual sense to the greatest extend, so that they can find parking spaces 
easily and find exits accurately. When people go into a certain area, they will feel a strong "magnet field effect", and then form a differentiated "city image" [5]. In the underground garage sign system of Chengdu New Century Global Center, theme graphics repeatedly and unified appear, and bring pleasant sensory experiences to drivers. They can strengthen the features of city image, and allow users to obtain basic understanding of the city. Thus, these theme graphics in garage can become the window of local culture and heritage.

Color Design. The difference of color collocation can bring different visual psychological experiences. The overall color collocation of the sign system will directly affect people's judgment and recognition of the parking space. Special experiments show that, when people enter a space, 75\% of their initial impressions come from the feeling of color; identification of shapes goes afterwards. [6]

Most underground garages are semi-closed. Because of the special environment, the selection of colors needs to be combined with specific lighting conditions. There are all kinds of pipelines on the top of this garage; considering the location of these pipelines, 3.4 meters storey height should be kept. White light with low color temperature is chosen as the optimized illumination method, in order to provide the garage with a safe, easy and reliable environment, and expand the space of the underground garage. According to the theme of each region, 8 neutral colors with warm, soft tone are selected to form a strong contrast to the fluorescent colors of warning signs, and prevent users from nervous experience.

Strengthening Guidance Information. From the underground garage to the main commercial center, consumers need to take the elevator. Signs on the inside and outside walls of the elevator, as well as the entrances and exits of the mall are most convenient ways to inform users about guidance information. Relevant information on underground garage should be put in the waiting area and inner space of elevators to clarify guiding information, and help users to remember through repetition.

Enhancing the coherence of information. Underground garage parking spaces are not fixed; drivers need to search for parking spaces in the underground garage. Therefore, underground garages have high demands on information integrity and information density. [7] When entering the garage, drivers need to control the driving speed, and select information to keep in mind. Continuous guiding information on walls, columns, roofs, floors and turning space of the garage can enhance the continuity of information and help drivers to form continuous memories.

Guiding information on walls and columns should be set according to the theme of each partition. Guiding information should also be provided in pedestrian area, safe passage, elevator space, entrances and exits of garage as well as the business area. Exposed pipelines ought to be colored according to the theme color of different safe partitions, in order to maintain the unity of the whole sign system. Guide facilities need to be designed and established in accordance with graphics and colors of different themes. Thus, each garage partition can have independent personality.

In the course of driving, hanging signs are most important source for drivers to identify directions. Therefore, hanging signs should be concise, understandable and contains appropriate information. Signs in different regions need to convey corresponding theme symbols. Combined with corresponding colors, these signs can remind drivers about their positions and make them to response accurately in the shortest period of time.

When pedestrians walking in and out of the elevator, guide information on the garage floor can effectively provide them with location information. Colors should be set according to parking information and the theme of partitions; clear colors and symbols should be painted on the ground of pedestrian area to remind clients. On the grounds of T-junctions and intersections, guidance information should be painted accord with information on hanging signs, in order to inform drivers about garage information.

\section{Foundation Design for the Sign System}

Theme pattern design. Eight different themes are designed for the underground garage of Chengdu New Century Global Center. On the basis of unique local elements, eight theme images are designed through art processing: hot pot; magic birds of Jinsha; dialect; leisure culture (covered 
bowl tea); national treasure, panda; ecological Chengdu; Sichuan Opera; hibiscus(Figure 2). These eight images reflect the inclusive, beautiful and comfortable life atmosphere of Chengdu from the perspectives of natural scenery and personal life. Eight obviously different colors are selected as theme color; theme patterns and English letters are combined together to identify specific garage partitions.
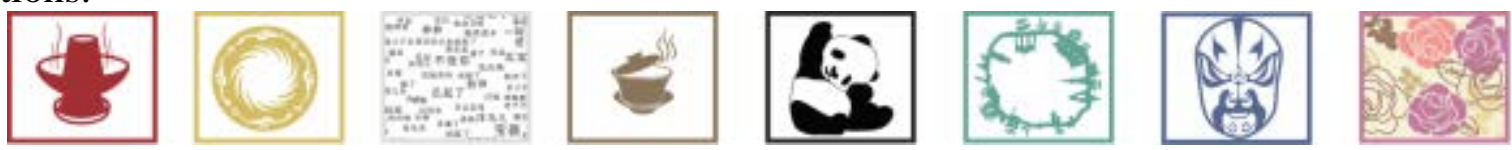

Fig.2 Theme patterns of underground garage in Chengdu New Century Global Center

Font design. The underground garage of Global Center uses English letters to realize partition; in each partition, numbers are used to mark each parking space. Font design needs to conform to visual aesthetic laws, and have distinctive visual images, in order to achieve the best effect of transferring literal message. [8]English letters should be easy to identify, and can eliminate people's insecurity feelings in underground space. The outer edges of English letters are processed into circular beads; people can feel relaxed and comfortable when reading these letters. (Figure 3)

\section{A B C DE F G H}

Fig.3 English letters of underground garage in Chengdu New Century Global Centre

Graphic symbol design. Graphic symbol is the carrier of information; it directly communicates with people, and guide people to receive information. Thus, the design of graphic symbols should be the considered as the main part of sign system design. [9] Through restructuring and transformation of basic graphs like circular, triangular and square patterns, basic elements of graphic symbols are selected. The combination of circular and square patterns give people a sense of balance, and eliminate their insecurity feelings in underground space, which greatly relaxes users and facilitates the guidance process. The colors of symbols are assigned according to the color of different partitions. (Figure 4)

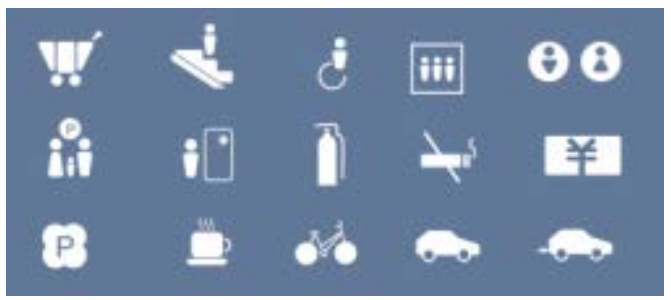

Fig.4 Graphic symbol of underground garage in Chengdu New Century Global Center

Designatedsymbol design. Designated symbols are the most important direction marks in the garage. Arrows can provide drivers with the sense of direction. Accurate placement and graphic symbols determine the efficiency of the whole system. The positions of signs should be set according to driver's visual fields, in order to achieve the purpose of accurate communication. On one hand, as a kind of visual symbol sign, designatedsymbols can indicate specific target objects; on the other hand, designated symbols are also an integral part of urban landscape. [10]

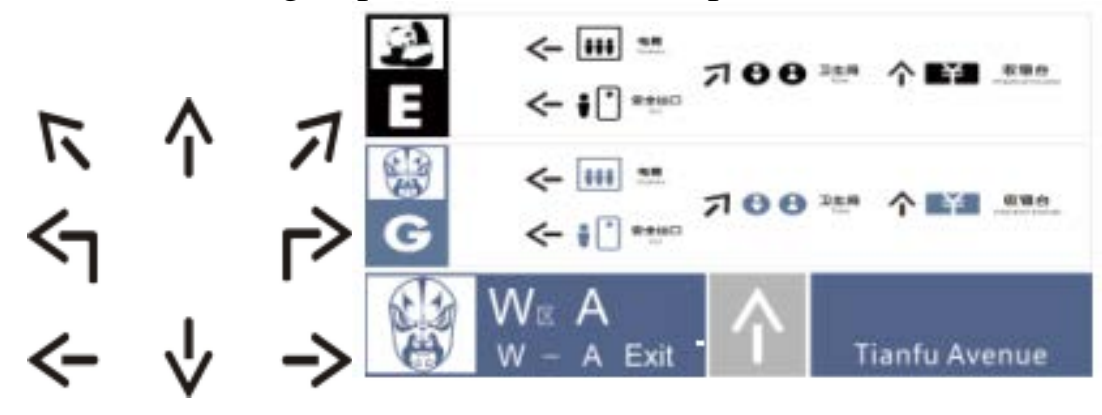

Fig.5 Designatedsymbol of underground garage in Chengdu New Century Global Center

With the continuous development of information interaction, sign system of underground garage in large-scale business districts can be combined with computer programs to realize data collection on parking information and personal location information. Users can use mobile phone to access 
information on parking spaces and return routes on the Internet, and realize convenient parking in underground garage.

\section{Conclusions}

Sign system design for underground garage in large commercial areas should not be limited in simple graphics in computers or floor plans. In addition to basic laws of garage sign system design, designers also need to consider regional culture patterns, users' behavior habits, and the actual environmental conditions. Details determine success or failure of the design. Theme patterns can help underground garage space to form unique characters, and attract people's attentions. Thus, guidance information of the garage can be remembered by users. This paper optimizes the existing sign system of the underground garage in Chengdu New Century Global Center, aiming to improve the comfort level of underground garage, represent unique local culture, and promote the image of Chengdu.

\section{Acknowledgement}

Fund Project: This paper is the outcome of the research, Design of Visual Sign System in Underground Parking Space, which is supported by Foundation for the Projects of Sichuan Landscape Architecture Center (Project No.: 2015YB05). It is also the outcome of The Project for Thesis Training Program in Sichuan Agricultural University (Project No.: 64009111).

\section{References}

[1] W. Shao, H.Y. Zhao, Visual recognition in the design of underground garage in large scale urban residential areas, J. Chinese and Overseas Architecture. 3 (2010) 73-75.

[2] J.L. He, B.Z. Chen, Static sign system in urban public spaces based on soty media information, J. Urban Planning Forum. 181 (2009) 58-63.

[3] X.R. Zheng, Application of sign information in commercial external space, J. Packaging Engineering. 36 (2015)128-131.

[4] J. Itten, Ding-yu Du (Trans.), The Art of Color, Shanghai World Book Publishing Company, Shanghai, 1999.

[5] H.Y. Zhang, Localization of cultural cognition of urban image, J. Urban Problems. 05 (2004) 6-11.

[6] B.H. Wang, L.L. Lin, Study on the design of color and environment of underground garage--take the underground garage of Puerta America hotel in Madrid, Spain as an example, J. Modern Decoration (Theory). 2 (2013) 87-88.

[7] L.J. Yu, X.D. Pan, The design of vehicle sign system in underground garage of city complex, J. Journal of Transportation Engineering and Information. 10 (2012)94-98.

[8] X.S. Liu, The importance of font design in graphic design, J. Packaging Engineering. 10 (2007) 233-235.

[9] Y. Bai, Design of graphic symbols in sign system. Wuhan University of Technology, 2006.

[10] X.C. Wu, Reflections on the design of the signage design of Xiamen Huandao Road, J. Art and Life. 6 (2013) 50-51. 